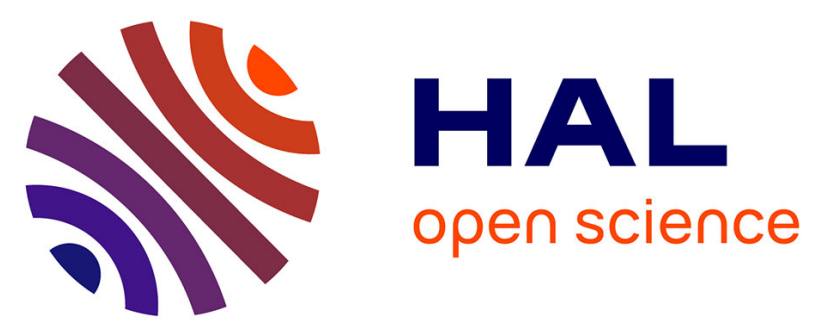

\title{
Performance Analysis of Network Coded Cooperation with Channel Coding and Adaptive DF-Based Relaying in Rayleigh Fading Channels
}

Xuan Thang Vu, Pierre Duhamel, Marco Di Renzo

\section{- To cite this version:}

Xuan Thang Vu, Pierre Duhamel, Marco Di Renzo. Performance Analysis of Network Coded Cooperation with Channel Coding and Adaptive DF-Based Relaying in Rayleigh Fading Channels. IEEE Signal Processing Letters, 2015, 22 (9), pp.1354-1358. 10.1109/LSP.2015.2405083 . hal-01120829

\section{HAL Id: hal-01120829 \\ https://hal.science/hal-01120829}

Submitted on 12 Mar 2015

HAL is a multi-disciplinary open access archive for the deposit and dissemination of scientific research documents, whether they are published or not. The documents may come from teaching and research institutions in France or abroad, or from public or private research centers.
L'archive ouverte pluridisciplinaire HAL, est destinée au dépôt et à la diffusion de documents scientifiques de niveau recherche, publiés ou non, émanant des établissements d'enseignement et de recherche français ou étrangers, des laboratoires publics ou privés. 


\title{
Performance Analysis of Network Coded Cooperation with Channel Coding and Adaptive DF-Based Relaying in Rayleigh Fading Channels
}

\author{
Thang X. Vu, Member, IEEE, Pierre Duhamel, Fellow, IEEE, and Marco Di Renzo, Senior Member, IEEE
}

\begin{abstract}
Network Coded Cooperation (NCC) is known to provide full diversity order and high spectral efficiency for uncoded cooperative networks. However, the understanding of NCC applied to signals that have been protected by some Forward Error Correction (FEC) codes is still limited. This letter analyzes the diversity order attainable by NCC with channel coding (Coded-NCC) in a network topology with multiple sources, one relay and in the presence of fast Rayleigh fading. Due to the difficulty of characterizing the exchange of information between the network decoder and the channel decoder, iterative network and channel decoding algorithms are usually studied with the aid of simulations. In this letter, we overcome this limitation by proposing a near-optimal receiver that performs network decoding and channel decoding in a single decoding step of an equivalent super code. An upper bound and a tight approximation of the Bit Error Rate (BER) for all sources are derived. Based on the upper bound, we analytically show that Coded-NCC achieves a diversity order equal to $2 f$, where $f$ is the minimum distance of the FEC code. This result generalizes those available for cooperative networks in the absence of channel coding (Uncoded-NCC), where the diversity order is equal to 2, as well as those available for coded transmission but without cooperation, where the diversity order is equal to $f$.
\end{abstract}

Index Terms-Convolutional codes, cooperative diversity, diversity order, network coding.

\section{INTRODUCTION}

Network Coded Cooperation (NCC) has recently gained attention because of the potential improvement in terms of diversity order and throughput that it can provide compared to conventional cooperative techniques [1]. In NCC, some network nodes, i.e., henceforth called relays, linearly combine multiple input packets and forward their combination either to the destination or to other relays. Therefore, the relays are capable of serving multiple sources in a single resource block, e.g., a time-slot. The spectral efficiency gain introduced by Network Coding (NC), as a result, may become more important in multiple-source networks. In [2], the capacity of a cooperative network with $\mathrm{NC}$ is evaluated and it is shown that a significant improvement with respect to classical relayaided networks is obtained. In [3], the diversity-multiplexing tradeoff and NCC is analyzed. Recently, the authors of [4] have derived the asymptotic Bit Error Rate (BER) of uncoded multiple-access relay networks, which provides insight onto the practical performance of NCC. The benefits introduced by

T. X. Vu is with the Singapore University of Technology and Design, Singapore. e-mail: xuanthang_vu@sutd.edu.sg.

P. Duhamel and M. Di Renzo are with the Laboratory of Signals and Systems (L2S, UMR 8506), CNRS - CENTRAL/SUPELEC - University ParisSud XI, France. e-mail: \{pierre.duhamel, marco.direnzo\}@1ss.supelec.fr.

Copyright (c) 2012 IEEE. Personal use of this material is permitted. However, permission to use this material for any other purposes must be obtained from the IEEE by sending a request to pubs-permissions@ieee.org.
NCC in multiple-source multiple-relay networks have recently been studied in [5]-[7]. These papers, however, do not take Forward Error Correction (FEC) coding into account, which is usually adopted in modern communication standards. The objective of the present paper is to address the achievable performance of NCC with channel coding (Coded-NCC).

Iterative Network and Channel Decoding (INCD) is an active field of research and various authors have investigated its BER performance. For example, the performance of binary NC with Convolutional Coding (CC) is studied in [8], [9] for propagation over quasi-static block fading channels. The generalization to non-binary $\mathrm{NC}$ is available in [10]. The interplay of NC and Low Density Parity Check (LDPC) coding is investigated in [11]. These papers, however, only resort to numerical simulations and do not provide any results about the achievable diversity order ${ }^{1}$. This mainly originates from the complexity of characterizing iterative decoding.

It is known that channel coding is capable of increasing the diversity order of non-cooperative networks for propagation over general block fading channels, i.e., the channel is fast varying and several channel gains are present in a codeword. More specifically, the diversity order depends on the number of channel gains in the codeword [13]. If channel coding is used in relay-aided wireless networks, the diversity order can be further increased. If relaying is replaced by NCC, the diversity order can, in principle, be increased even further. The objective of this letter is to mathematically characterize the achievable diversity order of Coded-NCC. In particular, we focus our attention on fully-interleaved (fast) Rayleigh fading channels, i.e., the channel gains change independently from symbol to symbol. This channel model can be seen as the limit of a general block fading channel model, where the number of channel gains is equal to the codeword length. In practice, this operating regime may be achieved with the aid of an ideal interleaver with infinite depth. Comments on the extension of this paper to other fading channels are provided in Section $\mathrm{V}$

An upper bound and a tight approximation of the BER for the sources are derived. Based on the upper bound, we analytically show that Coded-NCC achieves a diversity order equal to $2 f$, where $f$ is the minimum distance of the FEC code. This result generalizes those available for cooperative networks without channel coding (Uncoded-NCC), where the diversity order is equal to 2 [4], as well as those available for coded transmission but without cooperation, where the diversity order is equal to $f[14]$. Simulation results are used

\footnotetext{
${ }^{1}$ The diversity order is defined as the slope of the BER as a function of the Signal-to-Noise-Ratio (SNR) in a log-log scale [12].
} 
to verify the accuracy of the mathematical analysis.

The paper is organized as follows. Section II] describes the system model and the decoding algorithm. Section III analyzes the BER of Coded-NCC. Section IV] studies coding gain and diversity order. Finally, Section $\mathrm{V}$ concludes this paper.

\section{SySTEM MODEL}

Let us consider a cooperative network composed of $N_{s}$ sources $\mathcal{S}=\left\{\mathrm{S}_{1}, \ldots, \mathrm{S}_{N_{s}}\right\}$, one relay $\mathrm{R}$ and one destination D. All nodes are equipped with a single-antenna. The protocol is assumed to operate in orthogonal channels. For illustrative purposes, a Time Division Multiple Access (TDMA) protocol is considered. Perfect time synchronization is assumed. A cooperation phase consists of $N_{s}+1$ time-slots. In the first $N_{s}$ time-slots, each source broadcasts a data message to the relay and to the destination. In the $\left(N_{s}+1\right)$-th time-slot, the relay forwards a binary network-coded signal to the destination. A fast-fading channel model is assumed, i.e., the fading gains independently change from symbol to symbol.

\section{A. Processing at the Sources}

Let $\mathbf{u}_{i}, 1 \leq i \leq N_{s}$, be a message of length $K$ emitted by the source $\mathrm{S}_{i}$ in the time-slot reserved for its transmission, i.e., the $i$ th time-slot. The message $\mathbf{u}_{i}$ is encoded by using a CC with rate $K / N$, which provides a codeword $\mathbf{c}_{i}=\left\{c_{i, n}\right\}_{n=1}^{N}$ of $N$ coded bits. The codeword $\mathbf{c}_{i}$ is mapped, by using Gray coding, into a signal $\mathbf{x}_{i}=\left\{x_{i, l}\right\}_{l=1}^{L}$ that contains $L=N / \log _{2} M$ modulated symbols that belong to a $M$ Quadrature Amplitude Modulation ( $M$-QAM). The modulated codeword $\mathbf{x}_{i}$ is broadcasted to the relay and to the destination. Let $\mathbf{h}_{S_{i} R}=\left\{h_{S_{i} R, l}\right\}_{l=1}^{L}$ and $\mathbf{h}_{S_{i} D}=\left\{h_{S_{i} D, l}\right\}_{l=1}^{L}$ be the channel fading vectors of the links $\mathrm{S}_{i} \rightarrow R$ and $\mathrm{S}_{i} \rightarrow D$, respectively. The signals received at the relay and destination from the source $\mathrm{S}_{i}, 1 \leq i \leq N_{s}$, can be formulated as follows:

$$
\left\{\begin{array}{l}
y_{S_{i} R, l}=\sqrt{P_{S_{i} R}} h_{S_{i} R, l} x_{i, l}+z_{S_{i} R, l}, 1 \leq l \leq L \\
y_{S_{i} D, l}=\sqrt{P_{S_{i} D}} h_{S_{i} D} x_{i, l}+z_{S_{i} D, l}, 1 \leq l \leq L
\end{array}\right.
$$

where $P_{X Y}$, with $\mathrm{X} \in \mathcal{S}$ and $\mathrm{Y} \in\{\mathrm{R}, \mathrm{D}\}$, is the received signal power at node $\mathrm{Y}$ from node $\mathrm{X}$ including the path loss; $h_{X Y, l}$ is the channel gain of the channel $\mathrm{X} \rightarrow \mathrm{Y}$ corresponding to the $l$ th modulated symbol. $h_{X Y, l}$ is a complex Gaussian distributed random variable with zero mean and unit variance. From symbol to symbol, these random variables are statistically independent. $z_{(.)}$is the complex Gaussian distributed noise term with zero mean and variance $\sigma^{2}$.

\section{B. Processing at the Relay}

Upon receiving the $N_{s}$ packets from the sources, the relay applies the Bahl, Cocke, Jelinek and Raviv (BCJR) algorithm [20] to estimate the transmitted codeword $\mathbf{c}_{i}^{R}$ from the received vector $\mathbf{y}_{S_{i} R}$. Let $\Theta \triangleq\left\{S_{i} / \mathbf{c}_{i}^{R}=\mathbf{c}_{i}\right\}$ be the set of sources that successfully decode the transmitted codeword at the relay. By definition, $\Theta \subseteq \mathcal{S}$. In practice, the relay is capable of determining whether a codeword is received correctly or not with the aid of an error detecting code, e.g., by applying Cyclic Redundancy Check (CRC) decoding to the entire codeword. After applying the $\mathrm{CRC}$, the relay employs an Adaptive Decode-and-Forward (ADF) protocol for data transmission [15]. More specifically, the relay applies NC only to the messages that are successfully decoded, i.e., those belonging to $\Theta$. If $\Theta=\emptyset$, the relay remains silent. Let $\mathbf{c}_{R}=\left\{c_{R, n}\right\}_{n=1}^{N}$ denote the network-coded message at the relay. It can be formulated as follows: $c_{R, n}=\left\{\oplus c_{j, n}^{R}\right\}_{\mathrm{S}_{j} \in \Theta}$, where $\oplus$ denotes binary XOR operation. In this paper, XOR is chosen since it achieves full diversity order in single relay networks [7].

After $\mathrm{NC}$, the relay modulates $\mathbf{c}_{R}$ into the signal $\mathbf{x}_{R}=$ $\left\{x_{R, l}\right\}_{l=1}^{L}$ and forwards it to the destination. The signal received at the destination in time-slot $\left(N_{s}+1\right)$ is $y_{R D, l}=$ $\sqrt{P_{R D}} h_{R D, l} x_{R, l}+z, 1 \leq l \leq L$. It is worth mentioning that the relay needs to inform the destination of the messages that are successfully decoded at the relay. This is possible by adding $N_{s}$ bits of side information in the transmitted packet.

\section{Decoding at the Destination}

After receiving the $N_{s}+1$ packets from the sources and from the relay, the destination performs joint network/channel decoding. Let $\bar{\Theta}=\mathcal{S} \backslash \Theta$ denote the subset of messages from the sources that are not network-coded at the relay. By assuming that the channel state information of the relay-todestination links is available at the destination, the Maximum a Posteriori (MAP) demodulator can be formulated as follows:

$$
\begin{aligned}
& \widehat{\mathbf{u}}_{i}=\arg \max _{\mathbf{u}_{i}} \operatorname{Pr}\left\{\mathbf{u}_{i} \mid \mathbf{y}_{S_{i} D}\right\}, \mathrm{S}_{i} \in \bar{\Theta}, \\
& \widehat{\mathbf{U}}=\arg \max _{\mathbf{U}} \operatorname{Pr}\{\mathbf{U} \mid \mathbf{Y}\}, \mathrm{S}_{i} \in \Theta,
\end{aligned}
$$

where $\mathbf{U}=\left\{\mathbf{u}_{i_{1}}, \ldots, \mathbf{u}_{i_{|\Theta|}}\right\}$ is the collection of data messages belonging to $\Theta$, and $\mathbf{Y}=\left\{\mathbf{y}_{S_{i_{1}} D}, \ldots, \mathbf{y}_{S_{i_{\mid} \mid} D}, \mathbf{y}_{R D}\right\}$, with $|()$.$| denoting the cardinality of a set.$

As for the sources that are not network-coded at the relay, (2) can be solved by applying the BCJR algorithm. In particular, each source can apply it independently of the others. As for the sources that belong to $\Theta$, (3) might be solved by applying iterative decoding methods, as described in [9]. These methods, however, have two limitations: they are sub-optimal and they are not conveniently formulated for mathematical analysis and for identifying the achievable diversity order.

To overcome these issues, we look at the problem from a different point of view. In particular, (3) is interpreted as a "super code" whose input data is $\mathbf{U}$ and whose output data is $\mathbf{Y}$. The key idea is to interpret the signal transmitted from the relay as an additional parity bit (redundancy) that belongs to a super code, whose trellis representation is constituted by all the states of the individual trellises of the sources and that originate from using the network code at the relay. Therefore, both network and channel encoding/decoding are merged into a single encoding/decoding process that can be directly applied to the super code. If the channel code used by each source is $\mathbf{g}=\left[\begin{array}{ll}g_{1} & g_{2}\end{array}\right]_{1 \times 2}$ with rate $1 / 2$, for example, the super code $\mathbf{G}(\Theta)$ has a matrix form of size $|\Theta| \times 2(|\Theta|+1)$ that can be formulated as follows:

$$
\mathbf{G}(\Theta)=\left[\begin{array}{ccccccccc}
g_{1} & g_{2} & 0 & 0 & \ldots & 0 & 0 & g_{1} & g_{2} \\
\ldots & & & & & & & & \\
0 & 0 & 0 & 0 & \ldots & g_{1} & g_{2} & g_{1} & g_{2}
\end{array}\right] .
$$

With this interpretation at hand, (3) can (optimally) be solved by applying the BCJR algorithm to the super code $\mathbf{G}(\Theta)$, whose super codeword is $\mathbf{C}=\left[\mathbf{c}_{i_{1}}, \ldots, \mathbf{c}_{i_{|\Theta|}}, \mathbf{c}_{R}\right]$, 
where $\mathbf{c}_{R}=\left\{c_{R, n}\right\}_{n=1}^{N}$ and $c_{R, n}=c_{i_{1}, n} \oplus \ldots \oplus c_{i_{|\Theta|}, n}$. Since the trellis of the super code consists of all the combinations of the trellis states of the sources, the complexity of the proposed decoder exponentially increases with the number of sources $N_{s}$. Although it is more computationally intensive than the iterative decoding algorithms described in [9], it allows us to analyze the BER of Coded-NCC systems.

\section{PERformance AnAlysis}

Let $\mathcal{A}=a_{1} \ldots a_{N_{s}}$ with $a_{i} \in\{0,1\}$ be the $N_{s}$ bits of side information that are used for informing the destination of the sources that are network-coded at the relay. If the source $S_{i}$ is correctly decoded at the relay, $a_{i}=1$; otherwise, $a_{i}=0$. As a result, the successfully decoded set $\Theta$ can be better described by $\Theta_{\mathcal{A}}$. By definition, $\left|\Theta_{\mathcal{A}}\right|=\sum_{i=1}^{N_{s}} a_{i}$ and $\Theta_{0 \ldots 0}=\emptyset$. Let $q_{i} \triangleq \operatorname{Pr}\left\{\mathbf{c}_{i}^{R} \neq \mathbf{c}_{i}\right\}$ be the Frame Error Rate (FER) of the link $\mathrm{S}_{i} \rightarrow \mathrm{R}$. The probability of $\Theta_{\mathcal{A}}$, denoted by $p\left(\Theta_{\mathcal{A}}\right)$, is $p\left(\Theta_{\mathcal{A}}\right)=\prod_{i=1}^{N_{s}}\left(a_{i}\left(1-q_{i}\right)+\left(1-a_{i}\right) q_{i}\right)$. Let us denote $\bar{x} \triangleq \mathbb{E}\{x\}$ as the expectation of $x$, where the average is computed with respect to the fading channels. Since the links are assumed to be statistically independent, the BER of source $\mathrm{S}_{i}$ can be formulated as follows:

$$
\mathrm{BER}_{i}=\sum_{\Theta_{\mathcal{A}} / a_{i}=0} \bar{p}\left(\Theta_{\mathcal{A}}\right) \overline{\mathrm{Pe}}_{S_{i} D}+\sum_{\Theta_{\mathcal{A}} / a_{i}=1} \bar{p}\left(\Theta_{\mathcal{A}}\right) \overline{\mathrm{Pe}}_{\Theta_{\mathcal{A}}},
$$

where $\overline{\mathrm{Pe}}_{S_{i} D}$ is the average BER of the $\mathrm{S}_{i} \rightarrow \mathrm{D}$ link, which is independent of $\Theta_{\mathcal{A}}, \overline{\mathrm{Pe}}_{\Theta_{\mathcal{A}}}$ is the average BER of the source $\mathrm{S}_{i}$ that belongs to the successfully decoded set, which depends on $\Theta_{\mathcal{A}}$. Also, $\bar{p}\left(\Theta_{\mathcal{A}}\right)=\prod_{i=1}^{N_{s}}\left(\left(a_{i}\left(1-\bar{q}_{i}\right)+\left(1-a_{i}\right) \bar{q}_{i}\right)\right.$, where $\bar{q}_{i}$ is the average FER of the link $\mathrm{S}_{i} \rightarrow \mathrm{R}$.

Mathematical expressions of $\overline{\mathrm{Pe}}_{S_{i} D}$ can be computed as described in [14], since it is the average BER of classical CC. Upper bounds of the FER $\bar{q}_{i}$ of the $\mathrm{S}_{i} \rightarrow \mathrm{R}$ links can be obtained as described in [16], and they can be formulated as a function of the BER and of the codeword's length. The open issue is the computation of $\overline{\mathrm{Pe}}_{\Theta_{\mathcal{A}}}$.

\section{A. Computation of $\overline{\mathrm{Pe}}_{\Theta_{\mathcal{A}}}$}

$\overline{\mathrm{Pe}}_{\Theta_{\mathcal{A}}}$ is the BER of source $\mathrm{S}_{i}$ after performing the joint decoding that takes the super code $\mathbf{G}\left(\Theta_{\mathcal{A}}\right)$ into account. The successfully decoded set $\Theta_{\mathcal{A}}$ only contains $\left|\Theta_{\mathcal{A}}\right| \leq N_{s}$ sources, i.e., $\left\{\mathrm{S}_{i_{1}}, \ldots, \mathrm{S}_{i_{\left|\Theta_{\mathcal{A}}\right|}}\right\}$. We propose to compute $\overline{\mathrm{Pe}}_{\Theta_{\mathcal{A}}}$ by using the distance spectrum and the Pairwise Error Probability (PEP) of the super code $\mathbf{G}\left(\Theta_{\mathcal{A}}\right)$.

If $\mathbf{G}\left(\Theta_{\mathcal{A}}\right)$ is a CC, in fact, the BER of source $\mathrm{S}_{j} \in \Theta_{\mathcal{A}}$ can be formulated as $\overline{\mathrm{Pe}}_{\Theta_{\mathcal{A}}}=\sum_{d=F}^{+\infty} \mathrm{w}_{j}(d) \mathrm{P}_{\mathrm{u}}(d)$, where $F$ is the minimum distance of the super code $\mathbf{G}\left(\Theta_{\mathcal{A}}\right), w_{j}(d)$ is the input weight (i.e., the number of non-zero information bits) of source $\mathrm{S}_{j}$ and $\mathrm{P}_{\mathrm{u}}(d)$ is the Unconditioned PEP (UPEP) of receiving the super codeword with an output weight $d$ (i.e., the number of non-zero coded bits) and by assuming that the all-zero codeword has been transmitted. The main challenge of computing $\overline{\mathrm{Pe}}_{\Theta_{\mathcal{A}}}$ lies in determining the minimum distance $F$ of $\mathbf{G}(\Theta)$, the input weight $w_{j}(d)$ and how $d$ non-zero bits are distributed among the $\mathrm{S}_{j} \rightarrow \mathrm{D}$ and $\mathrm{R} \rightarrow \mathrm{D}$ links. Let us denote by $\mathbf{D}_{d}=\left\{d_{i_{1}}, \ldots, d_{i_{\Theta_{\mathcal{A}} \mid}}, d_{R}\right\}$ the weight pattern that specifies how $d$ weights are distributed among the $\mathrm{S}_{j} \rightarrow \mathrm{D}$ and $\mathrm{R} \rightarrow \mathrm{D}$ links, where $d_{j}$ is the output weight of the individual codeword transmitted via the channel $\mathrm{S}_{i_{j}} \rightarrow \mathrm{D}$ with $1 \leq j \leq\left|\Theta_{\mathcal{A}}\right|$ or via the channel $\mathrm{R} \rightarrow \mathrm{D}$. Then, $d=d_{i_{1}}+\ldots+d_{i_{\left|\Theta_{\mathcal{A}}\right|}}+d_{R}$. The input weight and the pattern can be computed via heuristic searching of the trellis of $\mathbf{G}\left(\Theta_{\mathcal{A}}\right)$. The following result is important for further analysis. Due to space limitation, its proof is omitted but it can be obtained as described in [17].

Lemma 1: For any successfully decoded set $\Theta_{\mathcal{A}} \neq \emptyset$, the minimum distance $F$ of the super code $\mathbf{G}\left(\Theta_{\mathcal{A}}\right)$ is equal to two times the minimum distance $f$ of the individual code g, i.e., $F=2 f$, and the pattern $\mathbf{D}_{F}=\left\{d_{i_{1}}, \ldots, d_{i_{\mid \Theta_{\mathcal{A}}} \mid}, d_{R}\right\}$ that corresponds to the minimum distance path of the super code has only two non-zero elements, i.e., $d_{j_{1}}=d_{j_{2}}=f, d_{j \neq j_{1}, j_{2}}=0$, with $j_{1}, j_{2}, j \in\left\{i_{1}, \ldots, i_{\left|\Theta_{\mathcal{A}}\right|}, R\right\}$.

With the aid of Lemma $1, \overline{\mathrm{Pe}}_{\Theta_{\mathcal{A}}}$ can be formulated as a function of the pattern $\mathbf{D}_{d}$, as follows:

$$
\overline{\mathrm{Pe}}_{\Theta_{\mathcal{A}}}=\sum_{d=F}^{+\infty} \sum_{\mathbf{D}_{d}} \mathrm{w}_{j}\left(\mathbf{D}_{d}\right) \mathrm{P}_{\mathrm{u}}\left(d \mid \mathbf{D}_{d}\right),
$$

where $\mathrm{P}_{\mathrm{u}}\left(d \mid \mathbf{D}_{d}\right)=\mathbb{E}\left\{\mathrm{P}_{\mathrm{c}}\left(d \mid \mathbf{D}_{d}\right)\right\}$ and $\mathrm{P}_{\mathrm{c}}\left(d \mid \mathbf{D}_{d}\right)$ is the Conditioned PEP (CPEP), which depends on the channel fading coefficients. In (6), it is assumed that an erroneous detected symbol can only be one of the nearest neighbor symbols. Since Gray mapping is assumed, a single bit error corresponds to a symbol error. Therefore, the CPEP $\mathrm{P}_{\mathrm{c}}\left(d \mid \mathbf{D}_{d}\right)$ can be approximated as follows [18]:

$$
\mathrm{P}_{\mathrm{c}}\left(d \mid \mathbf{D}_{d}\right)=\beta Q(\sqrt{2 \alpha \chi}),
$$

where $\chi=\sum_{j=i_{1}}^{i_{\left|\Theta_{\mathcal{A}}\right|} \mid} \sum_{k=1}^{d_{j}} \gamma_{S_{j} D, k}+\sum_{k=1}^{d_{R}} \gamma_{R D, k}, \gamma_{X Y, k}=$ $P_{X Y}\left|h_{X Y, k}\right|^{2} / \sigma^{2}$ is the instantaneous SNR of the link X $\rightarrow$ $\mathrm{Y}$ that is associated to the $k$ th signal symbol and $\alpha, \beta$ are some constants that depend on the $M$-QAM constellation. For example, $\beta=\alpha=1$ for 2 -QAM and $\beta=3 / 4, \alpha=2 / 5$ for 16-QAM. In Rayleigh fading channels, the instantaneous SNR $\gamma_{X Y, k}$ is an exponentially distributed random variable with average SNR equal to $\bar{\gamma}_{X Y, k}=\bar{\gamma}_{X Y}, \forall k$.

Upper Bound of $\mathrm{P}_{\mathrm{u}}\left(d \mid \mathbf{D}_{d}\right)$ : By applying the Chernoff bound to the Q-function defined as $Q(x) \leq \frac{1}{2} e^{-x^{2} / 2}$, we get:

$$
\begin{aligned}
& \mathrm{P}_{\mathrm{u}}\left(d \mid \mathbf{D}_{d}\right) \leq \frac{\beta}{2} \mathbb{E}\{\exp (-\alpha \chi)\} \\
& \quad \leq \frac{\beta}{2}\left(\alpha \bar{\gamma}_{R D}+1\right)^{-d_{R}} \prod_{j=i_{1}}^{i_{\left|\Theta_{\mathcal{A}}\right|}}\left(\alpha \bar{\gamma}_{S_{j} D}+1\right)^{-d_{j}},
\end{aligned}
$$

which originates from the fact that $\gamma_{S_{j} D, k}, \gamma_{R D, k}$ are statistically independent $\forall j, k$ and $\bar{\gamma}_{X Y, k}=\bar{\gamma}_{X Y}, \forall k$.

Approximation of $\mathrm{P}_{\mathrm{u}}\left(d \mid \mathbf{D}_{d}\right)$ : The Chernoff bound may not be very accurate for some system setups [13]. A better approximation may be obtained by formulating the Q-function as $Q(x) \simeq \frac{1}{12} \exp \left(-\frac{x^{2}}{2}\right)+\frac{1}{4} \exp \left(-\frac{2 x^{2}}{3}\right)$ [19. eq. (14)]. Then, $\mathrm{P}_{\mathrm{u}}\left(d \mid \mathbf{D}_{d}\right)$ can be approximated as follows:

$$
\begin{gathered}
\mathrm{P}_{\mathrm{u}}\left(d \mid \mathbf{D}_{d}\right) \simeq \frac{\beta}{12} \mathbb{E}\{\exp (-\alpha \chi)\}+\frac{\beta}{4} \mathbb{E}\{\exp (-4 \alpha \chi / 3)\} \\
\simeq \frac{\beta}{12}\left(\alpha \bar{\gamma}_{R D}+1\right)^{-d_{R}} \prod_{j=i_{1}}^{i_{\left|\Theta_{\mathcal{A}}\right|}}\left(\alpha \bar{\gamma}_{S_{j} D}+1\right)^{-d_{j}}+
\end{gathered}
$$




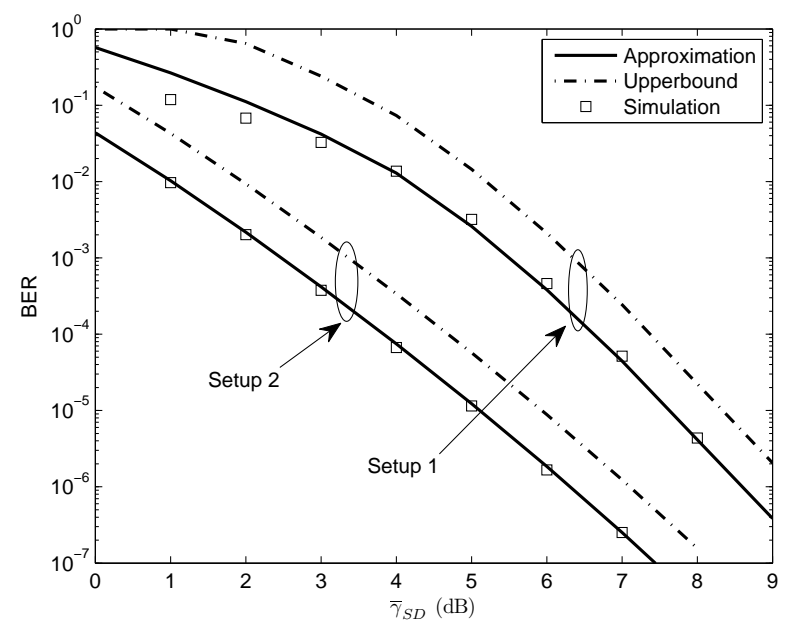

Fig. 1: Performance of Coded-NCC, $N_{s}=3$. Setup 1: $(\bar{\gamma}, \bar{\gamma}, \bar{\gamma})$, Setup 2: $(\bar{\gamma}, \bar{\gamma}+$ $12 \mathrm{~dB}, \bar{\gamma}+12 \mathrm{~dB})$.

$$
\frac{\beta}{4}\left(\frac{4 \alpha \bar{\gamma}_{R D}}{3}+1\right)^{-d_{R}} \prod_{j=i_{1}}^{i_{\left|\Theta_{\mathcal{A}}\right|}}\left(\frac{4 \alpha \bar{\gamma}_{S_{j} D}}{3}+1\right)^{-d_{j}}
$$

The result in (9) is obtained from the fact that the fading gains are independent. Therefore, the integral originating from $\chi$ in (7) can be factorized into the product of single integrals (of $\gamma_{X Y, k}$ ). By direct inspection of (8) and (9), we note that a diversity order equal to $d_{R}+d_{i_{1}}+\cdots+d_{i_{\Theta}}=d$ corresponds to $\mathrm{P}_{\mathrm{u}}\left(d \mid \mathbf{D}_{d}\right)$. By inserting (9) and (8) in (6) and (5), an upper bound and an approximation for the BER of the source $\mathrm{S}_{i}$ can be obtained. It is worth noting that, even though the output weight $d$ in (6) can be infinity, the BER is usually determined by a few first values of $d[14]$.

\section{B. Diversity Analysis for Coded-NCC}

Let us use the notation $X \stackrel{\circ}{=} \gamma^{-\eta}$ to state that $X$ has asymptotic diversity order $\eta$, where $\gamma$ is the average SNR. As for the direct links, $\bar{q}_{i} \stackrel{\circ}{=} \overline{\mathrm{Pe}}_{S_{i} D} \stackrel{\circ}{=} \gamma^{-f}$ for $1 \leq i \leq N_{s}$, where $f$ is the minimum distance of the individual code $\mathrm{g}$ [14]. Consequently, we obtain $p\left(\Theta_{\mathcal{A}}\right) \stackrel{\circ}{=} \gamma^{-f}$ if $\mathrm{S}_{i} \notin \Theta_{\mathcal{A}}$, and $p\left(\Theta_{\mathcal{A}}\right) \stackrel{\circ}{=}-\gamma^{-f}$ if $\mathrm{S}_{i} \in \Theta_{\mathcal{A}}$. The diversity order of $\overline{\mathrm{Pe}}_{\Theta_{\mathcal{A}}}$ is determined by the diversity order of $\mathrm{P}_{\mathrm{u}}\left(d \mid \mathbf{D}_{d}\right)$, since the input weight $\mathrm{w}_{j}\left(\mathbf{D}_{d}\right)$ is constant. From (6) and (8), it follows that $\overline{\mathrm{Pe}}_{\Theta_{\mathcal{A}}} \stackrel{\circ}{=} \gamma^{-2 f}$. Finally, from (5) we obtain $\mathrm{BER}_{i} \stackrel{\circ}{=} \gamma^{-2 f}$, from which we conclude that Coded-NCC achieves a diversity order that is two times larger than that of non-cooperative transmission with channel coding.

\section{Simulation Results}

All simulation results are obtained by assuming binary NC and Gray mapping. Each message consists of 512 bits. CodedNCC employs the CC [13 15] (in octal) with 1/2-rate and minimum distance equal to $f=6$. As benchmarks, four stateof-the-art transmission schemes are illustrated: Uncoded-NCC [4], non-cooperative transmission with channel coding, timesharing relaying, and INCD [8]-[10]. For a fair comparison, all schemes are assumed to have the same spectral efficiency of 3/4 bits per channel use. As a result, time-sharing relaying uses 8-QAM while for the other schemes 4-QAM is used. The first 6 values of the weights $d$ are used to compute the mathematical expression of the upper bound of the BER. For

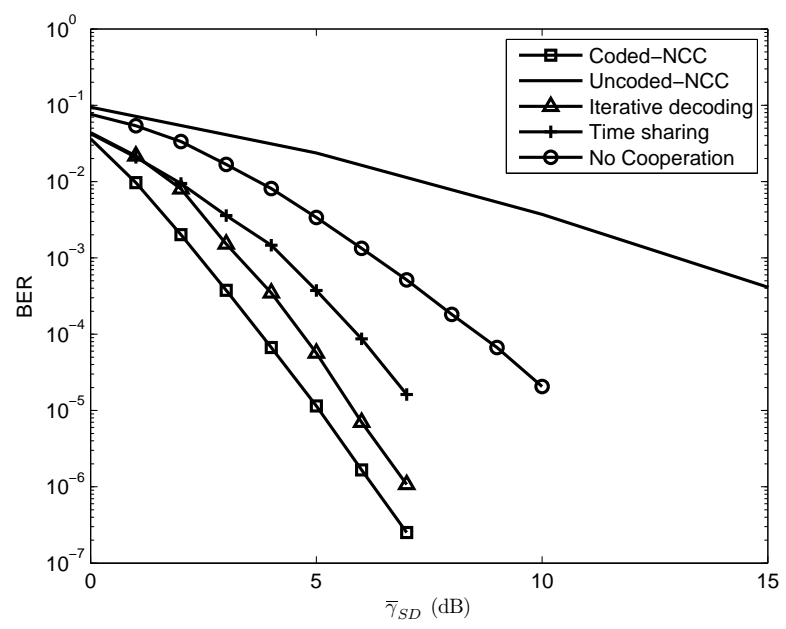

Fig. 2: BER comparison of Coded-NCC and state-of-the-art schemes, $\left(\bar{\gamma}_{S D}, \bar{\gamma}_{S R}, \bar{\gamma}_{R D}\right)=(\bar{\gamma}, \bar{\gamma}+12 \mathrm{~dB}, \bar{\gamma}+12 \mathrm{~dB})$.

simplicity, a symmetric network deployment is considered, i.e., $\bar{\gamma}_{S_{1} D}=\ldots=\bar{\gamma}_{S_{N_{s}} D}=\bar{\gamma}_{S D}$ and $\bar{\gamma}_{S_{1} R}=\ldots=\bar{\gamma}_{S_{N_{s}} R}=\bar{\gamma}_{S R}$.

Fig. 1 illustrates the performance of Coded-NCC for $N_{s}=$ 3. The proposed BER approximation is closely overlapped to the simulation results for both considered setups and for the SNR range of interest. In Setup 1, some gaps can be observed in the low SNR regime. This is due to the approximation of computing the FER of the source-to-relay links [16]. The upper bound overestimates the actual BER of approximately $1 \mathrm{~dB}$, but it provides the same diversity order as the approximation.

Fig. 2 compares the BER of Coded-NCC against the benchmarks. We observe that the proposed scheme outperforms the other schemes. In particular, Coded-NCC is capable of achieving a diversity order equal to 12 , while non-cooperative and Uncoded-NCC achieve a diversity order equal to 6 and to 2 , respectively. Furthermore, Coded-NCC performs $1 \mathrm{~dB}$ better than INCD and $2 \mathrm{~dB}$ better than time-sharing relaying at a BER of 1e-4. It is worth mentioning that, by using non-binary NC, the SNR gain between Coded-NCC and INCD may change but full diversity order is still guaranteed. Furthermore, we note that time-sharing relaying achieves the same diversity order as the non-cooperative scheme at low SNR. This is because the relay is not capable of successfully decoding the source message at low SNR. As the SNR of the source-to-relay links increases, however, time-sharing relaying is capable of achieving full diversity order, similar to Coded-NCC.

\section{CONCLUSION AND Discussion}

We have analyzed BER and diversity order of CodedNCC in fast Rayleigh fading channels. We have shown that a diversity order equal to two times that of the constituent channel code is achieved and it depends on the characteristics of the fading channel. In [17], it is shown, for example, that channel coding does not introduce any diversity gains in slow fading channels. In practical operating scenarios, the channel is usually in between slow fading and fast fading. In this case, the benefits of channel coding highly depend on the number of independent channels available in a codeword. The analysis of general block fading channels is postponed to future research work. 


\section{REFERENCES}

[1] R. Ahlswede, C. Ning, S. Y. R. Li, and R. W. Yeung, "Network information flow," IEEE Trans. Inf. Theory, vol. 46, no. 4, pp. 12041216, 2000.

[2] Z. Ding and K. Leung, "On the combination of cooperative diversity and network coding for wireless uplink transmissions," IEEE Trans. Veh. Technol., vol. 60, no. 4, pp. 1590-1601, 2011.

[3] C. Wang, M. Xiao, and M. Skoglund, "Diversity-multiplexing tradeoff analysis of coded multi-user relay networks," IEEE Trans. Commun., vol. 59, no. 7, pp. 1995-2005, 2011.

[4] A. Nasri, R. Schober, and M. Uysal, "Performance and optimization of network-coded cooperative diversity systems," IEEE Trans. Commun., vol. 61, no. 3, pp. 1111-1122, 2013.

[5] M. Di Renzo, M. Iezzi, and F. Graziosi, "On diversity order and coding gain of multisource multirelay cooperative wireless networks with binary network coding", IEEE Trans. Veh. Technol., vol. 62, no. 3, pp. 11381157, Mar. 2013.

[6] M. Di Renzo, M. Iezzi, and F. Graziosi, "Error performance and diversity analysis of multi-source multi-relay wireless networks with binary network coding and cooperative MRC", IEEE Trans. Wireless Commun., vol. 12, no. 6, pp. 2883-2903, June 2013.

[7] M. Di Renzo, "On the achievable diversity of repetition-based and relay selection network-coded cooperation", IEEE Trans. Commun., vol. 62, no. 7, pp. 2296-2313, July 2014.

[8] C. Hausl and P. Dupraz, "Joint network-channel coding for the multipleaccess relay channel," in IEEE 3rd Annual Communications Society on Sensor and Ad Hoc Communications and Networks (SECON), vol. 3, Sept 2006, pp. 817-822.

[9] X. T. Vu, M. D. Renzo, and P. Duhamel, "Multiple-access relaying with network coding: Iterative network/channel decoding with imperfect CSI," EURASIP J. Adv. Sig. Pr., no. 170, 2013.

[10] M. Hernaez, P. Crespo, and J. Del Ser, "On the design of a novel joint network-channel coding scheme for the multiple access relay channel," IEEE J. Sel. Areas Commun., vol. 31, no. 8, pp. 1368-1378, August 2013.

[11] Z. Guo, J. Huang, B. Wang, S. Zhou, J.-H. Cui, and P. Willett, "A practical joint network-channel coding scheme for reliable communication in wireless networks," IEEE Trans. Wireless Commun., vol. 11, no. 6, pp. 2084-2094, June 2012.

[12] M. Uysal, "Diversity analysis of space-time coding in cascaded rayleigh fading channels," IEEE Commun. Lett., vol. 10, no. 3, pp. 165-167, Mar 2006.

[13] R. Knopp and P. A. Humblet, "On coding for block fading channels," IEEE Trans. Inf. Theory, vol. 46, no. 1, pp. 189-205, 2000.

[14] A. Glavieux, Channel Coding in Communication Networks: From Theory to Turbo Codes. Wiley\& Sons Ltd, 2007.

[15] S. Ikki and M. Ahmed, "Performance analysis of adaptive decode-andforward cooperative diversity networks with best-relay selection," IEEE Trans. Commun., vol. 58, no. 1, pp. 68-72, 2010.

[16] M. Pursley and D. Taipale, "Error probabilities for spread-spectrum packet radio with convolutional codes and viterbi decoding," IEEE Trans. Commun., vol. 35, no. 1, pp. 1-12, 1987.

[17] X. T. Vu, M. D. Renzo, and P. Duhamel, "BER analysis of joint network/channel decoding in block rayleigh fading channels," in IEEE 24th International Symposium on Personal Indoor and Mobile Radio Communications (PIMRC), Sept 2013, pp. 698-702.

[18] J. G. Proakis and M. Salehi, Digital communications, 5th ed. Boston: McGraw-Hill Higher Education, 2008.

[19] M. Chiani, D. Dardari, and M. K. Simon, "New exponential bounds and approximations for the computation of error probability in fading channels," IEEE Trans. Wireless Commun., vol. 2, no. 4, pp. 840-845, 2003.

[20] L. Bahl, J. Cocke, F. Jelinek, and J. Raviv, "Optimal decoding of linear codes for minimizing symbol error eate (Corresp.)", IEEE Trans. Inf. Theory, vol. 20, no. 2, pp. 284-87, 1974. 